MIT-CTP-2345

NUB-3097-94TH

hep-ph/9408230

\title{
Discrete symmetries and isosinglet quarks in low-energy supersymmetry
}

\author{
Diego J. Castaño \\ Center for Theoretical Physics \\ Massachusetts Institute of Technology \\ Cambridge MA 02139 \\ and \\ Stephen P. Martin ${ }^{\dagger}$ \\ Department of Physics \\ Northeastern University \\ Boston MA 02115
}

\begin{abstract}
Many extensions of the minimal supersymmetric standard model contain superfields for quarks which are singlets under weak isospin with electric charge $-1 / 3$. We explore the possibility that such isosinglet quarks have low or intermediate scale masses, but do not mediate rapid proton decay because of a discrete symmetry. By imposing the discrete gauge anomaly cancellation conditions, we show that the simplest way to achieve this is to extend the $Z_{3}$ "baryon parity" of Ibáñez and Ross to the isosinglet quark superfields. This can be done in three distinct ways. This strategy is not consistent with grand unification with a simple gauge group, but may find a natural place in superstring-inspired models, for example. An interesting feature of this scenario is that proton decay is absolutely forbidden.
\end{abstract}

$\dagger$ Address after Sept. 1, 1994: Randall Physics Laboratory, University of Michigan, Ann Arbor MI 48109 
Extensions of the standard model with supersymmetry unbroken down to energies comparable with the electroweak-breaking scale can solve the naturalness problem associated with the Higgs scalar boson. It is remarkable that in the minimal supersymmetric standard model [1] (MSSM), the three gauge couplings appear to unify [2] at a scale $\sim 10^{16}$ $\mathrm{GeV}$, hinting at a grand unified theory (GUT) or some other organizing principle such as superstring theory. There is a potential phenomenological embarrassment in such theories, however; they contain chiral superfields for quarks which are singlets of weak isospin and carry electric charge $-1 / 3$. In GUT models, these isosinglet quarks necessarily appear in the same multiplets as the Higgs doublets of the MSSM, so that generically one might expect them to have masses comparable to the electroweak scale. In superstring-inspired models, the chiral superfields come from remnants of the $\mathbf{2 7}$ and $\overline{\mathbf{2 7}}$ representations of $E_{6}$. The masses of the isosinglet quark superfields are extremely model-dependent, and are typically determined by perturbations from flat directions in the superpotential. Therefore, again in superstring models, the isosinglet quarks can very often have low or intermediate scale masses.

The most general superpotential for the MSSM plus the isosinglet quarks is given schematically by:

$$
\begin{aligned}
W & =W_{0}+W_{1}+W_{2} \\
W_{0} & =Q H_{u} \bar{u}+Q H_{d} \bar{d}+L H_{d} \bar{e}+\mu H_{u} H_{d}+\mu_{D} D \bar{D} \\
W_{1} & =Q L \bar{D}+\bar{u} \bar{e} D \\
W_{2} & =Q Q D+\bar{u} \bar{d} \bar{D} .
\end{aligned}
$$

Here $Q, \bar{u}, \bar{d}, L, \bar{e}$ are the quark and lepton chiral superfields of the MSSM; $H_{u}, H_{d}$ are the Higgs doublet chiral superfields of the MSSM; and $D, \bar{D}$ are the chiral superfields for the isosinglet quarks. Under the gauge group $S U(3)_{c} \times S U(2)_{L} \times U(1)_{Y}$, they transform as

$$
\begin{aligned}
& Q \sim(\mathbf{3}, \mathbf{2}, 1 / 6) \quad \bar{u} \sim(\overline{\mathbf{3}}, \mathbf{1},-2 / 3) \quad \bar{d} \sim(\overline{\mathbf{3}}, \mathbf{1}, 1 / 3) \\
& L \sim(\mathbf{1}, \mathbf{2},-1 / 2) \quad \bar{e} \sim(\mathbf{1}, \mathbf{1}, 1) \quad H_{u} \sim(\mathbf{1}, \mathbf{2}, 1 / 2) \\
& H_{d} \sim(\mathbf{1}, \mathbf{2},-1 / 2) \quad D \sim(\mathbf{3}, \mathbf{1},-1 / 3) \quad \bar{D} \sim(\overline{\mathbf{3}}, \mathbf{1}, 1 / 3) .
\end{aligned}
$$

For now, we assume the conservation of the usual $Z_{2}$ matter parity which is given for each chiral superfield in the MSSM by $(-1)^{3(B-L)}$, where $B$ and $L$ are the usual baryon number 
and total lepton number. This matter parity is trivially related to R-parity by a minus sign for fermions. Thus $Q, \bar{u}, \bar{d}, L, \bar{e}$ all have matter parity -1 , and $H_{u}, H_{d}, D, \bar{D}$ each have matter parity +1 . Later we will consider the implications of relaxing this assumption. We assume that there are 3 chiral families of quarks and leptons, and the isosinglet quark superfields $D$ and $\bar{D}$ may or may not also be replicated, but we suppress all flavor and gauge indices.

The most pressing phenomenological problem posed by the existence of isosinglet quarks is the possibility of rapid proton decay. For example, if both of the terms $Q L \bar{D}$ and $Q Q D$ existed in the superpotential with couplings of order unity, and $D, \bar{D}$ had a mass $\mu_{D}$ in the $\mathrm{TeV}$ range, then the proton would decay in minutes due to one-loop diagrams with the virtual exchange of an isosinglet quark and a wino. The dominant decay mode would be $p \rightarrow K^{+} \bar{\nu}$, for which Kamiokande has established the experimental limit [3] $\tau\left(p \rightarrow K^{+} \bar{\nu}\right)>10^{32}$ yrs. More generally, the presence of either term in $W_{1}$ together with either term from $W_{2}$ will prevent us from consistently assigning $B$ or $L$ to the chiral superfields in the theory, generically resulting in catastrophic proton decay. If the isosinglet quarks exist at all, then there appear to be two ways out of this disaster; either $D$ and $\bar{D}$ must be very heavy so that their effects on low energy physics very nearly decouple, or some additional symmetry must be invoked to explain why either $W_{1}$ or $W_{2}$ or both are missing.

Both of these potential solutions are problematic in a supersymmetric GUT. It is possible to arrange for $D$ and $\bar{D}$ to obtain a very large mass; however, this requires some cleverness because at least one copy of $D$ and $\bar{D}$ lives in the same multiplet of the GUT gauge group as $H_{u}$ and $H_{d}$. Various proposals have been put forward to effect a separation in mass scales between $D, \bar{D}$ and $H_{u}, H_{d}$, including the "sliding singlet" mechanism [4], the "missing partner" mechanism [5], the related "missing VEV" mechanism [6], and Higgses as Nambu-Goldstone bosons [7]. These attempts generally require an intricate system of global symmetries to provide for realistic quark and lepton masses. It is also possible in supersymmetric GUTs to accept light isosinglet quarks but to rely on delicate cancellations among couplings to prevent proton decay [8]. 
In this paper we consider instead the possibility that isosinglet quarks $D, \bar{D}$ are light, but a discrete symmetry prohibits the terms from either $W_{1}$ or $W_{2}$ or both. This strategy is not consistent with a GUT based on a simple gauge group, but could be useful in superstring-inspired models, for example. The possibilities may then be divided into three cases, as follows:

Case A: $Q L \bar{D}$ and $\bar{u} \bar{e} D$ are allowed; $Q Q D$ and $\bar{u} \bar{d} \bar{D}$ are forbidden. Then we can assign baryon number and lepton number $B=1 / 3, L=1$ to $D$ and $B=-1 / 3, L=-1$ to $\bar{D}$. Thus $D$ and $\bar{D}$ are "leptoquarks".

Case B: $Q Q D$ and $\bar{u} \bar{d} \bar{D}$ are allowed; $Q L \bar{D}$ and $\bar{u} \bar{e} D$ are forbidden. Then we can assign baryon number and lepton number $B=-2 / 3, L=0$ to $D$ and $B=2 / 3, L=0$ to $\bar{D}$. Thus $D$ and $\bar{D}$ are "diquarks".

Case C: $Q Q D, \bar{u} \bar{d} \bar{D}, Q L \bar{D}$, and $\bar{u} \bar{e} D$ are all forbidden. Here it is not yet clear how to assign $B$ and $L$ to the isosinglet quark superfields, since they have no renormalizable superpotential interactions other than a mass term.

There has already been much interest [9-15] in the phenomenological implications of each of these three cases, particularly in the context of superstring models based on remnants of $E_{6}$. In this paper we will examine possibilities for discrete symmetries which can enforce the missing couplings in each of the three cases. Specifically, we consider a $Z_{N}$ symmetry under which each chiral superfield transforms as

$$
\Phi \rightarrow \exp \left(2 \pi i \alpha_{\Phi} / N\right) \Phi
$$

where the $\alpha_{\Phi}$ are the additive $Z_{N}$ charges. An operator is allowed if and only if the sum of its $Z_{N}$ charges is $0[\bmod N]$. For simplicity, we will assume that the $Z_{N}$ charges are not family-dependent; this seems to be required for the quark superfields anyway in order to allow for the observed Cabibbo-Kobayashi-Maskawa mixing. Since even a small violation of the discrete symmetry could result in catastrophic proton decay, it is strongly suggested that the $Z_{N}$ is a "gauged" discrete symmetry. One way [16] (but perhaps not the only way) to obtain a gauged discrete symmetry is to break a gauged $U(1)$ symmetry with an 
order parameter whose charge is $N q$, where the smallest non-zero $U(1)$ charge assignment in the theory is $q$. Unlike a global symmetry, a gauged discrete symmetry is automatically protected against violation by Planck-scale and other non-perturbative effects. As shown in [17-20], such gauged discrete symmetries are subject to stringent requirements based on anomaly cancellation.

Requiring that the $Z_{N}$ symmetry allow the usual Yukawa couplings and masses in $W_{0}$, one immediately obtains some relations between the $Z_{N^{-}}$charges. Thus

$$
\alpha_{H_{d}}=-\alpha_{H_{u}} \quad \text { and } \quad \alpha_{\bar{D}}=-\alpha_{D}
$$

are required in order to allow for Higgs and isosinglet quark masses respectively and

$$
\alpha_{\bar{u}}=-\alpha_{Q}-\alpha_{H_{u}}, \quad \alpha_{\bar{d}}=-\alpha_{Q}+\alpha_{H_{u}}, \quad \alpha_{\bar{e}}=-\alpha_{L}+\alpha_{H_{u}}
$$

in order to allow for Yukawa couplings. Each of these equations is understood to hold modulo $N$. We get further constraints $[\bmod N]$ in each of the three cases:

Case A: $\quad \alpha_{D}=\alpha_{Q}+\alpha_{L}$ and $3 \alpha_{Q}+\alpha_{L} \neq 0$

Case B: $\quad \alpha_{D}=-2 \alpha_{Q}$ and again $3 \alpha_{Q}+\alpha_{L} \neq 0$.

Case C: $\quad \alpha_{D} \neq \alpha_{Q}+\alpha_{L}$ and $\alpha_{D} \neq-2 \alpha_{Q}$

Now, cases A and B are in some sense more interesting, because in case $\mathrm{C}$ the isosinglet quarks are relatively sterile, having only gauge interactions with the chiral superfields of the MSSM. Therefore, we consider cases A and B first.

To further constrain the $Z_{N}$ symmetry, we now consider the discrete anomaly cancellation conditions of Ibáñez and Ross [17]. First we consider the mixed $Z_{N} \times S U(n) \times S U(n)$ anomaly cancellation condition, which is given in general by

$$
2 \sum_{i} \alpha_{i} T_{i}=0 \quad[\bmod N]
$$

where $T_{i}$ is the $S U(n)$ Dynkin index for each representation, normalized so that the fundamental representation has $T=1 / 2$. By plugging in the constraints already considered, 
it is easy to show that the $Z_{N} \times S U(3)_{c} \times S U(3)_{c}$ anomaly always cancels. However, the $Z_{N} \times S U(2)_{L} \times S U(2)_{L}$ anomaly cancellation condition is given by

$$
n_{f}\left(3 \alpha_{Q}+\alpha_{L}\right)=0 \quad[\bmod N]
$$

where $n_{f}=3$ is the number of chiral MSSM families. This is quite non-trivial, since we found that in both cases A and B,

$$
3 \alpha_{Q}+\alpha_{L} \neq 0 \quad[\bmod N]
$$

in order to allow only the appropriate interactions for $D$ and $\bar{D}$. The only way to satisfy both of these constraints simultaneously is to take $N$ to be a multiple of 3 , since that is the number of families. While larger values of $N$ certainly might give interesting models, we will take $N=3$ in the following for simplicity; it is worth noting that larger discrete symmetries are harder to obtain in superstring models.

The mixed gravitational $\left(Z_{N} \times G \times G\right)$ anomaly cancellation condition is given in general by

$$
\sum_{i} \alpha_{i}=0 \quad \begin{aligned}
{[\bmod N] } & (N \text { odd }) \\
{[\bmod N / 2] } & (N \text { even }) .
\end{aligned}
$$

This gives no further constraint for $N=n_{f}=3$. Finally we consider the $Z_{N}^{3}$ anomaly cancellation condition, which is given in general by

$$
\sum_{i} \alpha_{i}^{3}=r N+\eta s N^{3} / 8
$$

where $\eta=0(1)$ for $N$ odd (even) and $r, s$ are integers, and for $N=3, r$ must in fact be a multiple of 3 [17]. Given the relations already found, the cubic anomaly then cancels provided that $\alpha_{H_{u}}=-\alpha_{L}$. The $Z_{N}^{3}$ anomaly cancellation condition actually can always be satisfied for any choice of charge assignments [19], but in our case this would require the existence of heavy fields with fractional $Z_{3}$ charges, so that the underlying discrete symmetry would have to be at least as large as $Z_{9}[20]$.

Now, one can always take $\alpha_{Q}=0$, by redefining the $Z_{N}$ symmetry according to

$$
\alpha_{\Phi} \rightarrow \alpha_{\Phi}+6 n Y_{\Phi}
$$


where $n$ is an appropriate integer and $Y_{\Phi}$ is the weak hypercharge of $\Phi$. From eq. (5), $\alpha_{L}$ cannot vanish, so we can also choose $\alpha_{L}=1$ without loss of generality. In our case, this completely fixes all of the $Z_{3}$ charges. For the chiral superfields of the MSSM, we have

$$
\alpha_{Q}=0 ; \quad \alpha_{L}=1 ; \quad \alpha_{\bar{u}}=1 ; \quad \alpha_{\bar{d}}=-1 ; \quad \alpha_{\bar{e}}=1 ; \quad \alpha_{H_{u}}=-1 ; \quad \alpha_{H_{d}}=1
$$

For the isosinglet quark superfields there are now three possibilities, which in fact just correspond to the three cases described above:

Case A: $\quad \alpha_{D}=1 ; \quad \alpha_{\bar{D}}=-1$,

Case B: $\quad \alpha_{D}=0 ; \quad \alpha_{\bar{D}}=0$,

Case C: $\quad \alpha_{D}=-1 ; \quad \alpha_{\bar{D}}=1$

Thus we have uniquely fixed the charges of the chiral superfields under the discrete symmetries $Z_{3}^{A}$ and $Z_{3}^{B}$ for cases $\mathrm{A}$ and $\mathrm{B}$ respectively, and found a $Z_{3}^{C}$ for case $\mathrm{C}$ whose MSSM charge assignments are consistent with cases A and B. [Of course, in case C only, $N$ need not be a multiple of 3 since (5) need not hold. If one were only interested in the sterile $D, \bar{D}$ of case $\mathrm{C}$, one could have a consistent solution for any $N$ by, e.g., assigning $Z_{N}$ charges $\alpha_{D}=-\alpha_{\bar{D}}=1$ and $\alpha_{i}=0$ for all MSSM superfields.]

Note that each of $Z_{3}^{A}, Z_{3}^{B}$, and $Z_{3}^{C}$, when restricted to the fields of the MSSM, give nothing other than the discrete "baryon parity" proposed by Ibáñez and Ross [18] as an alternative to the usual matter parity, and which is given in their classification scheme by $R_{3} L_{3}$. They showed that this is the only family-independent $Z_{3}$ discrete symmetry with the minimal particle content which satisfies the discrete anomaly cancellation conditions. Its reappearance here is not at all surprising, since the existence of the isosinglet quarks cannot affect the anomaly cancellation conditions, due to the constraint that $D$ and $\bar{D}$ must have opposite charges to allow a mass term in the superpotential.

It is natural to consider the extension of this model to include gauge-singlet neutrino chiral superfields $\bar{\nu}$ in order to provide for a realistic and non-trivial neutrino mass spectrum via the seesaw mechanism [21]. Thus we may consider adding to the usual superpotential 
terms of the form

$$
W_{\text {seesaw }}=L H_{u} \bar{\nu}+M_{\bar{\nu}} \bar{\nu} \bar{\nu}
$$

Both of these terms are allowed if we assign $\alpha_{\bar{\nu}}=0$. Then the anomaly cancellation conditions are not affected, and the term $\bar{\nu} \bar{d} D$, which is naturally associated with $W_{1}$, is allowed in case $\mathrm{A}$ and forbidden in cases $\mathrm{B}$ and $\mathrm{C}$.

In the scenario described here, the usual $Z_{2}$ matter parity which is equivalent to $\mathrm{R}$ parity is actually optional. If one does not impose matter parity, the superpotential might a priori include $B$-violating terms

$$
W_{3}=\bar{u} \bar{d} \bar{d}
$$

and $L$-violating terms

$$
W_{4}=Q L \bar{d}+L L \bar{e}+L H_{u}
$$

However, $Z_{3}^{A, B, C}$ necessarily forbid $\bar{u} \bar{d} \bar{d}$, so that there can be no $B$ violation among renormalizable interactions. In fact, the $Z_{3}$ charges as given above are equal, for each chiral superfield, to $B-2 Y$ where $Y$ is the weak hypercharge. Since $Y$ is conserved, $Z_{3}^{A, B, C}$ invariance implies the selection rule

$$
\Delta B=0 \quad[\bmod 3]
$$

for operators of arbitrarily high dimension! Therefore, proton decay is absolutely forbidden by $Z_{3}^{A, B, C}$. Likewise, neutron-anti-neutron oscillations are absolutely forbidden by this same selection rule, since they would violate $B$ by 2 units. Soft supersymmetry-breaking interactions do not affect this conclusion, because they respect the same symmetries as the terms in the superpotential. Although $B$-violating effects are highly constrained by the selection rule (7), baryogenesis can still occur if a lepton number asymmetry is partially transformed into a baryon number asymmetry by high temperature electroweak effects.

Note that $\left\langle H_{u}\right\rangle$ and $\left\langle H_{d}\right\rangle$ transform non-trivially under $Z_{3}^{A, B, C}$, producing the spontaneous symmetry breaking

$$
S U(2)_{L} \times U(1)_{Y} \times Z_{3}^{A, B, C} \rightarrow U(1)_{E M} \times Z_{3}^{A, B, C}
$$


at the electroweak scale. The $Z_{3}^{\prime A, B, C}$ charges are given for each particle by $B+Q_{E M}$, where $Q_{E M}$ is the electric charge. Thus $B$ violation is still governed by the selection rule (7), as should be clear from the fact that the $H_{u}$ and $H_{d}$ carry no baryon number.

On the other hand, all of the lepton-number violating interactions in $W_{4}$ are allowed by the discrete symmetries $Z_{3}^{A, B, C}$. If R-parity is imposed in addition to the $Z_{3}^{A, B, C}$ symmetry, then $L$ will be conserved for all renormalizable interactions except $W_{\text {seesaw. Also, }}$. imposing R-parity will forbid mixing between $\bar{d}$ and $\bar{D}$ in case $\mathrm{A}$, which may be important in avoiding flavor-changing neutral currents. If R-parity is not imposed in addition to $Z_{3}^{A, B, C}$, there will be dramatic effects on accelerator searches for supersymmetry, since the LSP may decay into leptonic states inside the detector, and sleptons will have direct two-body decays into lepton+neutrino.

The fermionic components of $D, \bar{D}$ have a Dirac mass, $\mu_{D}$. Their scalar partners will also receive contributions to their masses from soft supersymmetry-breaking terms. The isosinglet quarks of cases A and B can readily decay to MSSM states. In case A, a possible decay signature for the scalar leptoquark is a jet and either a lepton or missing energy. The heavier of the scalar and fermionic leptoquark can also decay to the lighter and a neutralino [11]. The fermionic leptoquark can also decay semileptonically to two- or threebody states, depending on the kinematics. In case B, the direct scalar diquark two-jet decay will be difficult to see because of comparatively large QCD backgrounds [12,15]. The fermionic diquark may decay through interesting two- or three- body channels, depending on the sparticle mass spectrum.

In case $\mathrm{C}$, there is a phenomenological danger from the fact that the isosinglet quarks cannot decay into MSSM particles and might therefore be too stable. If R-parity is not conserved, there is an additional renormalizable interaction available to the isosinglet quarks in Case C, namely

$$
[\bar{u} \bar{D} \bar{D}]_{F}
$$

which seems to imply the curious assignment $B=-1 / 6$ and $L=0$ for $D$, although one could also assign $B=4 / 3, L=0$ to $D$, so that (8) violates $B$ by 3 units in agreement with 
the selection rule (7). This operator is forbidden by $Z_{3}^{A}$ and $Z_{3}^{B}$, and it vanishes unless there is more than one copy of $\bar{D}$. In any case, it does not help the lightest of the isosinglet (s)quarks to decay. If it is forbidden, then the renormalizable interactions do not fix $B$ or $L$ for the isosinglet quarks. There is a possible non-renormalizable operator which can allow the isosinglet quarks to decay, namely the dimension-6 operator

$$
[\bar{u} \bar{d} \bar{d} \bar{d} D]_{F}
$$

This is in fact the only operator of dimension 6 or less which is allowed by $Z_{3}^{C}$ and which can provide for isosinglet (s)quarks decaying into standard model states. If this provides the dominant decay mode for the isosinglet quarks, then we are led to the assignments $B=4 / 3$, $L=0$ for $D$ in case C. This dimension- 6 operator is suppressed by two powers of some putative high mass scale $M_{X}$, and leads to a many-body decay with lifetime $\sim M_{X}^{4} / \mu_{D}^{5}$. So the case $\mathrm{C}$ isosinglet quark may be long-lived and cosmologically dangerous if $M_{X} \gg \mu_{D}$, although this depends crucially on the nature of the new physics at the scale $M_{X}$, about which we will not speculate here. Also, it is conceivable that particles not considered here which are lighter than $D, \bar{D}$ might provide additional decay channels in case C.

Of course, the presence of light or intermediate scale $D, \bar{D}$ will affect the running of the gauge couplings, in general ruining the unification of gauge couplings and washing out the successful "prediction" of $\sin ^{2} \theta_{W}$. However, the addition of other thresholds (due to vectorlike particles at intermediate scales) might easily restore the correct value of $\sin ^{2} \theta_{W}$, perhaps with a true gauge coupling unification scale which is higher than the apparent unification scale at $\sim 10^{16} \mathrm{GeV}$. Realistic superstring models often have several such intermediate thresholds. As we have already mentioned, the strategy used in this paper is not consistent with a GUT. This is true even if one takes the GUT multiplet partners of the MSSM Higgs to be very heavy and does not identify them with $D$ and $\bar{D}$, because the $Z_{3}$ charges of the MSSM quark and lepton superfields are not consistent with assigning them to GUT multiplets.

Cancellation of discrete anomalies might also occur in superstring models through a discrete version of the Green-Schwarz (GS) mechanism [22]. This may occur if the axion 
which is the partner of the dilaton transforms non-trivially under the discrete gauge group. However, the discrete GS mechanism does not allow any new solutions to the constraints given above in the usual case that the Kac-Moody levels $k_{2}$ and $k_{3}$ of the gauge groups $S U(2)_{L}$ and $S U(3)_{c}$ are equal. To see this, suppose we have a discrete $Z_{N}$ symmetry which satisfies (2) and (3). With a discrete GS mechanism, the $Z_{N} \times S U(2)_{L} \times S U(2)_{L}$ and $Z_{N} \times S U(3)_{c} \times S U(3)_{c}$ mixed anomaly cancellation conditions become

$$
\begin{aligned}
n_{f}\left(3 \alpha_{Q}+\alpha_{L}\right) & =2 \delta_{\mathrm{GS}} k_{2} & & {[\bmod N] } \\
0 & =2 \delta_{\mathrm{GS}} k_{3} & & {[\bmod N] }
\end{aligned}
$$

respectively, where $\delta_{\mathrm{GS}}$ is a constant. But now from (11) we immediately find that if $k_{3}=k_{2}$, then (10) just reduces to the previous constraint (4).

There have been previous attempts to implement discrete symmetries to remove or suppress proton decay from light color triplets in superstring-inspired models. However, these preceded the appearance of [17] and so do not take into account discrete anomaly cancellation. Reference [9] considers a $Z_{2}$ symmetry which is somewhat similar to our case A, but which has a $Z_{2} \times S U(2)_{L} \times S U(2)_{L}$ anomaly, and also a $Z_{2}$ symmetry which is discrete anomaly-free and corresponds to our case C, but which requires massless neutrinos. They also consider a family-dependent $Z_{3}$ symmetry intended to suppress $\mu \rightarrow e \gamma$, but which has a $Z_{3} \times S U(2)_{L} \times S U(2)_{L}$ anomaly. In references [10] and [13], other $Z_{2}$ discrete symmetries corresponding to our cases $\mathrm{A}$ and $\mathrm{B}$ are proposed, but they have $Z_{2} \times S U(2)_{L} \times$ $S U(2)_{L}$ anomalies, as we have shown on general grounds.

In this paper, we have found that light weak-isosinglet quark superfields can be added to the particles of the MSSM without causing rapid proton decay, if the theory is invariant under a discrete symmetry. By using the discrete gauge anomaly cancellation conditions, we showed that by far the most economical way to do this is to extend the "baryon parity" of [18] to the isosinglet quark superfields. In fact, each of the three possible ways to do this can lead to acceptable phenomenology. Note that in general, one can have isosinglet quarks for each of cases $\mathrm{A}, \mathrm{B}$, and $\mathrm{C}$ peacefully coexisting, since the restrictions of $Z_{3}^{A, B, C}$ to the MSSM chiral superfields are consistent. The three classes of isosinglet quarks will not mix 
with each other, since they have different $Z_{3}$ charges. Even if there are no isosinglet quarks near the $\mathrm{TeV}$ scale, the $Z_{3}$ symmetry may be useful for preventing proton decay in models with $D, \bar{D}$ at intermediate scales. An intriguing theoretical aspect of the $Z_{3}$ symmetry is that its consistency is tied to the presence of 3 chiral families in the MSSM through the discrete anomaly cancellation conditions. Finally, we note that the scenario described here is falsifiable, since the selection rule (7) forbids all proton decay. Future proton decay searches at Super Kamiokande and ICARUS will therefore be crucial tests.

Acknowledgments: We are grateful to Pierre Ramond for helpful comments. The work of D.J.C. was supported in part by the U.S. Department of Energy under cooperative agreement DE-FC02-94ER40818 and in part by the Texas National Research Laboratory Commission under grant RGFY93278C. The work of S.P.M. was supported in part by the National Science Foundation grants PHY-90-01439 and PHY-93-06906 and U. S. Department of Energy grant DE-FG02-85ER40233.

\section{References}

1. For reviews, see H. P. Nilles, Phys. Rep. 110, 1, (1984) and H. E. Haber and G. L. Kane, Phys. Rep. 117, 75, (1985).

2. P. Langacker, in Proceedings of the PASCOS90 Symposium, Eds. P. Nath and S. Reucroft, (World Scientific, Singapore 1990) J. Ellis, S. Kelley, and D. Nanopoulos, Phys. Lett. 260B, 131, (1991); U. Amaldi, W. de Boer, and H. Furstenau, Phys. Lett. 260B, 447, (1991); P. Langacker and M. Luo, Phys. Rev. D44, 817, (1991).

3. Kamiokande collaboration, K. S. Hirata et. al., Phys. Lett. B220, 308, (1989).

4. E. Witten, Phys. Lett. B105, 267, (1981); L. E. Ibáñez and G. G. Ross, Phys. Lett. B110, 215, (1982).

5. B. Grinstein, Nucl. Phys. B206, 387, (1982); A. Masiero, D. V. Nanopoulos, K. Tamvakis, and T. Yanagida, Phys. Lett. B115, 380, (1982).

6. S. Dimopoulos and F. Wilczek, preprint NSF-ITP-82-07 (unpublished); K. S. Babu 
and S. M. Barr, Phys. Rev. D48, 5354, (1993).

7. K. Inoue, A. Kakuto, and T. Takano, Prog. Theor. Phys. 75, 664, (1986); A. Anselm and A. Johansen, Phys. Lett. B200, 331, (1988); R. Barbieri, G. Dvali, and A. Strumia, Nucl. Phys. B391, 487, (1993).

8. M. J. Bowick and P. Ramond, Phys. Lett. 131B, 367, (1983).

9. M. Drees and X. Tata, Phys. Rev. Lett. 59, 528, (1987).

10. Y. Kizukuri, Phys. Lett. B185, 183, (1987).

11. V. Barger, N. G. Deshpande, and K. Hagiwara, Phys. Rev. D36, 3541, (1987).

12. V. D. Angelopoulos, J. Ellis, H. Kowalski, D. V. Nanopoulos, N. D. Tracas, and F. Zwirner, Nucl. Phys. B292, 59, (1987).

13. E. Ma, Phys. Rev. Lett. 60, 1363, (1988).

14. E. Ma and D. Ng, Phys. Rev. D39, 1986, (1989).

15. J. L. Hewett and T. G. Rizzo, Phys. Rep. 183, 193, (1989).

16. L. Krauss and F. Wilczek, Phys. Rev. Lett. 62, 1221, (1989).

17. L. E. Ibáñez and G. G. Ross, Phys. Lett. B260, 291, (1991).

18. L. E. Ibáñez and G. G. Ross, Nucl. Phys. B368, 3, (1992).

19. T. Banks and M. Dine, Phys. Rev. D45, 1424, (1992).

20. L. E. Ibáñez, Nucl. Phys. B398, 301, (1993).

21. M. Gell-Mann, P. Ramond, and R. Slansky, in Sanibel Talk, CALT-68-709, Feb 1979, and in Supergravity, (North Holland, Amsterdam, 1979; T. Yanagida, in Proc. of the Workshop on Unified Theories and Baryon Number in the Universe, Tsukuba, Japan, 1979, edited by A. Sawada and A. Sugamoto (KEK Report No. 79-18, Tsukuba, 1979).

22. M. B. Green and J. H. Schwarz, Phys. Lett. B149, 117, (1984). 